\title{
MEMORIAS ESCOLARES EN CONTEXTOS DE CRISIS: REFLEXIONES EDUCATIVAS SOBRE MOMENTOS SIGNIFICATIVOS DE LA HISTORIA ARGENTINA EN EL CONTEXTO DEL BICENTENARIO
}

\author{
José Alberto Tranier*
}

\begin{abstract}
RESUMEN: El presente artículo intentará llevar adelante una revisación crítica de ciertos momentos claves que incidieron en el rumbo y accionar de la vida pedagógica y educativa en nuestro país. Para tal fin, habremos de trabajar a partir de algunos relatos actuales provenientes de personas que transitaron diferentes momentos de nuestra historia en la escuela primaria: bajo la dictadura de la década del 1970; bajo el primer gobierno peronista; y tras la crisis del 2001. De esta manera, relatos y memorias mediarán de hilo conductor de nuestra trama argumentativa haciendo especial referencia en primer lugar a la educación y la dictadura; en segundo, a la escuela y el Peronismo, para luego finalizar haciendo alusión a la educación y la crisis del 2001; ya que, algo que quizá podamos ir adelantando, es que el núcleo fuerte común de todos aquellos relatos es que, aún al día de hoy, se resisten a estar ausentes o caer en el olvido.
\end{abstract}

Palabras claves: Memorias. Relatos. Escuela. Historia. Crisis.

\section{SCHOOL MEMORIES IN CONTEXT OF CRISIS: EDUCATIONAL REFLECTIONS ON SIGNIFICANT MOMENTS IN THE HISTORY OF Argentina in the context of the Bicentennial}

ABSTRACT: In this work we will try to make a critical reflection on some important issues regarding the history of our country, Argentina, which have influenced the development of the pedagogical and educational life in our country. In order to do that, we will work with different stories and memories from people who attended primary school in different periods of our history: under dictatorship in the 1970's; under the first Peronist government; and after the 2001 crisis. Those stories and memories are important to us because they will let us learn about some educational singularities related to what those people felt and lived on those historical moments at school. They also conduct our argumentation, which makes special reference to: education and

Escuela de Ciencias de La Educación de la Universidad Nacional de Rosario (UNR). Rosario - Argentina.

Contato com o autor: <jtranier@gmail.com>

Educ. Soc., Campinas, v. 35, n. 127, p. 511-527, abr.-jun. 2014 
dictatorship; education and Peronism; education and the 2001 crisis. Moreover, something that can also be said about those memories is that the strong common feature to all of them, even now, is that they resist being effaced or forgotten.

Key words: Memories. Stories. School. History. Crisis.

MÉMOIRES SCOLAIRES DANS LES CONTEXTES DE CRISE: RÉFLEXIONS ÉDUCATIVES DES MOMENTS SIGNIFICATIFS DE L'HISTOIRE ARGENTINE DANS LE CONTEXTE DU BICENTENAIRE

RÉSUMÉ: L'article se propose à faire une révision critique de quelques moments-clés qui ont été importants dans les directions et les actions de la vie pédagogique et éducative de notre pays. Pour cela, nous avons travaillé à partir de quelques témoignages actuels venants des personnes qui ont parcouru différents moments de notre histoire à l'école primaire: sous la dictature des années 1970; sous le premier gouvernement Péroniste; et après la crise de 2001. De cette façon, les témoignages et les mémoires serviront comme le fil conducteur de notre argumentation, en faisant tout d'abord référence spéciale à l'enseignement et à la dictature; en suite à l'école et au Péronisme, pour juste après se consacrer à l'allusion à l'enseignement et à la crise de 2001. Tout cela pour justifier que le point fort en commun de tous ces témoignages est que, même dans nos jours, ils se réfusent d'être absents ou même à se faire oubliés.

Mots-clés: Mémoires. Témoignages. Ecole. Histoire. Crise.

\section{Introducción}

$\mathrm{L}$

o aquí trabajado forma parte de una reflexión originada por la celebración del primer bicentenario de la República Argentina y se basa en algunas argumentaciones provenientes de los (hoy ya adultos) alumnos que transitaron sus pasos en la escuela primaria en tres circunstancias históricas significativas a nivel político. Importa aquí distinguir o hacer la salvedad de que, más allá de las diferencias constitutivas inherentes a su correspondiente época, el énfasis de este trabajo estará puesto más en dar cuenta de los efectos en el orden de la producción de la subjetividad de aquellos niños bajo aquellos períodos (y que aún al día de hoy perduran); que en compartir o elaborar en forma exhaustiva un contexto histórico político de las condiciones sociales y de los aciertos o desventuras educativas de aquellas coyunturas.

Con lo anterior no se busca impugnar el valor histórico ni proponer un abordaje "liviano", sino centrarnos en un aspecto poco trabajado y el cual nos remite necesariamente a los modos de producción de subjetividad que incidieron en la labor pedagógica y en la producción del deseo de los alumnos - esto es, las formas de habitar lo escolar bajo aquellos períodos históricos. 
El orden de presentación no es casual ni caprichoso. El lector podrá darse cuenta que no están regidos por la linealidad histórica, sino que ha sido sustentado mediante la propia percepción e identificación de la idea fuerza más persistente, predominante, que perduraba en la memoria de los relatos y éste, entonces, ha sido el criterio privilegiado con el cual decidimos exponer nuestro trabajo: la educación y la dictadura; escuela y el Peronismo; y la crisis del 2001.

Los instrumentos que nos posibilitaron llevar adelante esta propuesta de trabajo han sido los relatos de vida, las entrevistas semi-estructuradas, las biografías escolares, empleadas a partir de un diseño de trabajo hermenéutico-crítico, puesto que, a través del análisis crítico como una herramienta teórica imprescindible al momento de significar, indagar e interpretar aquello que se desprende en forma natural y "espontánea" a partir de los relatos, será enmarcado dentro de nuestros propósitos de trabajo. Esto es, la indagación de sentidos e ideologías instaladas en los mismos, pero no concebidas como entidades subjetivas o intersubjetivas, sino como resultantes de procesos de subjetivación inherentes al accionar de estructuras objetivas de cada coyuntura histórica.

Hermenéutico, entonces, porque se propone interpretar sentidos y reconstruir el sistema de significaciones que subyacen a los relatos; crítico, porque intenta trascender el plano de lo visto y oído, de lo inmediato, para interiorizarse en profundidad, y de este modo, llegar al trasfondo de donde se acuñan los sentidos y que, para nosotros, es el de las condiciones sociales, histórico-educativas, culturales, económicas y políticas de los modos de producción de subjetividad percibidos en aquellos relatos. Comenzaremos a abordar, pues, el primer punto, referente a los relatos sobre la educación y la dictadura.

\section{Educación y dictadura}

Y porque en la historia de los hombres cada acto de destrucción encuentra su respuesta, tarde o temprano, en un acto de creación. (Eduardo Galeano)

Intentar dar cuenta, entonces, de ciertas conceptualizaciones históricas en relación con la educación y a la última dictadura, en nuestro país, no es una tarea que resulte sencilla. Sobre todo, porque a pesar del paso del tiempo y de las continuas revisiones históricas críticas, hay huellas dolorosas en el orden de la subjetividad que aún se resisten a considerarse agotadas. Por el contrario, creemos que demandan en forma constante una reflexión por parte de toda la comunidad y de la sociedad. Los relatos aquí presentados, en relación con la educación y el régimen dictatorial, 
nos permiten inferir o dar cuenta acerca de cómo se constituyen y perciben las similitudes y diferencias en torno a ese espacio simbólico socioeducativo en las propias escuelas, a partir de de aquel 24 de Marzo de 1976.

La conmemoración del primer Bicentenario como Nación, que tuvo lugar el 25 de mayo del 2010, brindó el marco preciso para revisar e interrogar (nos) acerca de qué modelo de país - y de sociedad - estamos construyendo por medio de la educación: qué voces han sido escuchadas a lo largo de nuestra historia, puestas en primer plano; o de qué nos hablan aquellas otras más tenues y muchas veces silenciadas, en esta suerte de polifonía "institucional" que se replica, pliega y despliega a lo largo de estas dos veces centenarias Nación. Si en la coyuntura presente debemos atribuirle una "función" a la educación, la misma tendrá necesariamente que estar ligada con la unión y con la cohesión de los pueblos; pero no solamente como metáfora de "autoconservación" sino como posibilidad de reconocimiento de aquel otro sujeto que me interpela; y en donde su mirada, entonces, se significa como un llamado de responsabilidad de uno mismo para con el otro, que convoca a la acción. Como sostiene Filloux (2008, p. 151): "en el corazón de lo pedagógico es lo relacional lo que está en juego y el orden pedagógico no puede ser sino un orden de libertad, excluyente de toda violencia que perturbe el libre desarrollo del niño".

\section{"Hoy tiran papelitos, mañana tiran bombas..."}

Sin embargo, y ya centrándonos en el primer apartado sobre "educación" y "dictadura", la abrupta separación de muchos de los docentes que evidenciaban diferencias políticas e ideológicas, hacia el nuevo régimen de organización nacional a lo largo de muchas escuelas de nuestro país, fue vivenciada por gran parte de los alumnos como algo violento, inesperado y fuera de toda lógica. Nos detendremos especialmente en el relato de un ex-alumno de la escuela modelo "Constancio C. Vigil"1 de la ciudad de Rosario, República Argentina; todo un emblema de la capacidad de lucha de una institución por haber intentado instituir nuevas formas de relacionamiento entre la sociedad y la educación.

A través de los relatos compartidos, no es de extrañar que aún se puedan percibir ciertas sensaciones y emociones que dejan entrever la presencia de un dolor el cual, al día de hoy, pareciera que no se resigna a ser tan fácilmente olvidado. Lo anterior nos da la pauta de que, por un lado, la existencia de aquellas formas de relacionamiento pedagógico, centrados a su vez en otras maneras propuestas e acceder al conocimiento (talleres literarios, de poesía, teatro, música, fuerte vínculo entre la escuela y su comunidad), confirma que la capacidad de llevar a cabo nuevos actos de institución (instituyentes) creadores de nuevos sentidos han forjado, hasta el día de hoy, huellas indelebles en la memoria de aquellos alumnos. 
Pero lamentablemente, por el otro, que aquel accionar embanderado en un nuevo estandarte educativo tuvo que ser necesariamente silenciado y negado en toda su capacidad crítica de transformación. Importa aquí, entonces, compartir algunos fragmentos que parecieran recuperar, a la luz de su memoria, un compromiso con dar cuenta de aquello acontecido:

[...] a pesar de que era chico, me acuerdo que, casi de un día para otro, empezaron a cambiar todas las maestras, inclusive la mía. Lo que pasó, es que ella era militante de una organización - por supuesto de esto me enteré más adelante - y un papá de un compañero mío también pertenecía a la misma organización ${ }^{2}$ y compartían (maestra y papá de mi compañero) militancia juntos, y solían conversar en los recreos, siempre juntos, conversaban más de lo habitual que con un papá cualquiera, yo me daba cuenta de eso, de que conversaban seguido, y siempre los observaba desde el patio de la escuela, durante los recreos, ya que él siempre traía a su hijo quien era, como dije, compañero mío. La cuestión es que absolutamente de un día para otro, no los vimos más. Ni al papá de mi amigo, ni a la señorita. Desaparecieron.

Lo anterior podría ser pensado en función de la lógica o coherencia interna de aquel proceso de reorganización nacional. Ya que, al igual que afirma Tedesco (1993, p. 9): "una fase del intento autoritario, la más obvia y externa, consistió en expulsar docentes, controlar contenidos, controlar las actividades de los alumnos y sus padres y regular los comportamientos visibles (ropa, cortes de pelo, etc.)". De esta manera, la exposición de este relato aún conserva la fuerza en su memoria de ciertas huellas que operaron como signos exteriores de "visibilidad" para el ejercicio - y puesta en práctica - del poder disciplinario y sus formas de retórica o participación en el espacio institucional.

Desde una perspectiva similar, Kaufmann y Doval (1997, p. 48) aluden a un discurso del Presidente de Facto, Videla, quien expresaba que: "[...] debe quedar claro que los hechos acaecidos el 24 de marzo, no materializan solamente la caída de un gobierno. Significan, por el contrario, el cierre definitivo de un ciclo histórico y la apertura de uno nuevo, cuya característica estará dada por la tarea de reorganizar la Nación".

Debido a ello es que pensamos que reorganizar la Nación incluía, obviamente, también reorganizar la escuela. El recuerdo de este hoy ya adulto prosigue contándonos que:

[...] de ese grupo de maestras que vinieron, nuevas casi todas de ellas, la gran mayoría eran autoritarias; inclusive a compañeros míos, los que se portaban mal, la maestra que sustituyó a la anterior desaparecida - lo tomaba de la oreja y lo sacaba del salón... también recuerdo que una vez estábamos todos hablando y, jugando, tiramos un avioncito de papel por el aire. Entonces se enojó mucho y con voz y mirada odiosa, nos retó y gritando dijo: “¡Basta! hoy tiran papelitos, mañana tiran bombas...”. 
Esto nos permite adherir a lo expresado por Tedesco (1987, p. 38) cuando sostiene que:

[...] la congruencia entre los diferentes fragmentos del bloque de poder tuvo como objetivo disciplinar la sociedad, lo cual, pedagógicamente, puede producirse como restauración del orden, las jerarquías y la autoridad. En este sentido, el nuevo modelo se presentó como una revalorización del autoritarismo pedagógico tradicional.

De allí la percepción que permitió a los alumnos identificar ciertos rasgos o patrones reiterativos, configuradores del ethos y del ser docente requerido para esos tiempos, traducidos en procesos de interpelación - y de mutuo reconocimiento - de las docentes con una ideología afín al proceso de facto de "reorganización nacional". El relato continúa con la propia percepción del entrevistado acerca de la noción de autoridad percibida en el marco de la escuela:

Tampoco me voy a olvidar más de la Directora nueva que habían puesto: vestía toda de negro, absolutamente toda de negro, incluso le decíamos "Morticia”", y encima tenía el pelo largo hasta la cintura... nos daba miedo, estupor, sin saber qué pasaba, sin entender bien... pero toparse con ella era lo peor que nos podía pasar... nos daba mucho miedo.

Hannah Arendt, en clara referencia a las diversas posiciones teóricas coexistentes acerca del tratamiento y concepción del pasado histórico, sostiene que éste "escapa" a la posibilidad real de concebirlo como objeto de estudio de "dominación"; esto es, aquella idea que expresa la necesidad de "dominarlo". Más aún, rechaza firmemente aquellos tópicos vulgares y enfáticamente pasa a afirmar que: "Lo mejor que puede lograrse (con el pasado) es saber con precisión qué fue, soportar este conocimiento y luego aguardar y ver qué resulta de este conocimiento y del hecho de soportarlo" (ARENDT, 2008, p. 30).

Quizás, a la luz de ese grupo de pequeños niños que no comprendían bien qué sucedía pero que soportaban - e incorporaban en forma de conocimiento -, las condiciones de sometimiento a través del dispositivo pedagógico planteado por la dictadura, los significantes ligados al sentido de negritud del color dado por la vestimenta, por el pelo y por la apariencia funcionaron como una suerte de tramitación simbólica capaz de "explicarle" a ellos "algo" sobre la idea de la muerte, "algo" sobre la desaparición de su maestra, o "algo" del papá de su compañero; o sobre el terror que se iniciaba. En ese contexto, la figura de la máxima autoridad escolar disponible para ellos - la "nueva" directora - así vestida, "misteriosamente" aparecida de "la nada", ofrecía las condiciones necesarias para depositar y mitigar una densa incertidumbre de la cual los niños no estaban ajenos, y quizás, también, como una forma de iniciar un camino de simbolización acerca de algo de aquel horror que pronto habría de cobrarse la vida de más de treinta mil desaparecidos. 


\title{
La escuela y el Peronismo
}

Situándonos ahora en este breve recorrido - y selección - histórica, el primero de los siguientes relatos remite a un hombre de 65 años, quien pasó gran parte de su escolaridad primaria bajo las dos presidencias del Peronismo. De allí que decidimos entablar un diálogo para que pueda contarnos acerca de qué es aquello que recuerda de su paso por la escuela primaria:

\begin{abstract}
Si tengo que hacer memoria, en forma inmediata me acuerdo de que en la escuela primaria, en 1953, en el año anterior muere Evita, entonces al año siguiente se eligió en la escuela un busto en su memoria pagado por la cooperadora. La presidenta cooperadora era la mamá de un compañero mío, entonces siempre vestía con una foto de Evita una cinta de luto, un ramillete, una foto de Perón, la bandera argentina, y siempre estaba en todos los actos escolares y siempre nos hablaba y comparaba a Sarmiento, San Martín, Belgrano con Perón. Al año siguiente - después del homenaje - cae Perón y entonces estaba prohibido en todos lados nombrarlo, silbar la marcha, (Decreto n. 4.161/56 de Aramburu); todo estaba prohibido, la sigla CGT, Evita, etc. Había pasado un año que, junto con otros compañeros, se nos ocurrió ir a hablar con la mamá de nuestro amigo - quien seguía al mando de la cooperadora - sin que supiera la directora, ya que sabíamos que no habría de permitirlo, con el fin de intentar recuperar el busto de Evita que se "rumoreaba" que aún estaba en un sótano de la escuela y que, próximamente, se lo habría de destruir. Fui a su casa, toco a su puerta, la saludo y le pregunto si se acordaba de mí, que era el amigo de su hijo. Por supuesto que se acordaba y allí en el umbral de su puerta le cuento lo que queríamos hacer y a medida que escuchaba de qué se trataba la invitación, se le fue transformando la cara, se enojó y gritando para que todos escuchen me echó diciendo: ¡No sé de qué busto me habla! y cómo puede venir a mi casa pensando que "la cooperadora" podría admitir siquiera hacer algo que vaya en contra de nuestra querida escuela...! A partir de ese día, aprendí a que en la escuela tenía que "aparentar" una cosa para que no me reten, y guardarme mi propio "pensar" para no tener problemas, por lo menos, en esos años en donde todavía, era niño.
\end{abstract}

Continuando con estos fragmentos de memorias, ahora es el turno de una mujer de 65 años perteneciente a otra escuela, pero que nos brinda una percepción diferente de su paso por la primaria bajo aquella época histórica:

Cuando se fue Perón, yo tenía 10 años, o sea que viví todos los primeros diez años de mi vida con en el Peronismo. Lo que yo te puedo decir es que, para mí, ha sido una experiencia muy fea porque mi papá no era peronista. Y bueno, eso significaba que a mi papá lo echaban siempre de los trabajos por no ser peronista, él trabajaba de linotipista y trabajaba en el diario La Capital de acá de Rosario. Yo me acuerdo muy bien que por no afiliarse, no lo dejaban trabajar, te aclaro que no era industrial, no éramos ricos, por el contrario, mi papá era pobre, era obrero pero tenía sus ideales. Así que jamás se afilió y tuvo que trabajar en forma clandestina en imprentas que le pagaban menos de la mitad de lo que pagarían si estaba afiliado; pero trabajaba mucho para que podamos ir a la escuela. Ya casi en los últimos años de Perón, en cada manzana, había un jefe de manzana, peronista, que sabía, de esa manzana, quiénes eran y quienes no peronistas. La cuestión es que el jefe de donde yo vivía, justo era el esposo de mi señorita en la escuela quien, yo notaba que siempre hacía diferencia con los otros compañeros e, implícitamente, me 
miraba como que "sabía" de aquel "secreto" familiar y entonces yo me sentía como que estaba siempre en falta, por lo menos, en lo concerniente a la escuela.

La indagación de la memoria conlleva un esfuerzo por tratar de contener ciertas marcas de un sufrimiento interior el cual, a la luz del relato, se revela como un pasado que despierta. En cierto sentido, se cumple aquello expresado por Ricoeur (2009, p. 87) cuando sostiene que: "Cada uno de nosotros se identifica con la historia que puede relatar sobre sí mismo...".

\begin{abstract}
Después que falleció Evita, nos obligaban a que todos los niños nos pongamos un moñito negro, te imaginarás que mi papá no quería que lo lleve. Le dijo a mi mamá: "ella no va a llevar ningún moño porque no se le murió ningún familiar". Entonces me mandaban sin el moño. La primera vez que entré, la directora revisaba a los niños a ver si todos llevaban, cuando me ve a mí la directora me dice: "señorita, pase a Dirección... se puede saber por qué no ha traído el moñito?". Entonces le dije, inocentemente la verdad: "lo que pasa que mi papá no quiso que lo trajera"... "Ah sí?, muy bien, se queda toda la mañana en dirección y mañana o viene con el moñito puesto, o se presenta con su papá, que ya vamos a hablar con él". Mientras tanto, con ocho años, estuve todo el día arrinconada en la dirección. Cuando llegué a mi casa, llorando en un baño de lágrimas, mi mamá que quería la tranquilidad de la familia, para que no sufra y para que no le pase nada a mi papá, y sin que él se entere, me hizo un moñito que lo escondía en un bolsillo; entonces, salía de la escuela sin el moñito, cuando estaba por llegar, me lo ponía y cuando me iba, me lo volvía a sacar... pero, te imaginas la responsabilidad que era para mí, con ocho años, llevar, sacar el moño, y la culpa que me daba por mi papá que no sabía... Gracias al "moñito", por lo menos la maestra y la directora me trataban mejor... Lo interesante es que tras la caída de Perón, tuve que llevar algo a la dirección y los cuadros de Perón a caballo, otro de Evita de luto, un busto de cada uno ellos, ya no estaban. Entonces, inocentemente, sorprendida ya que aún seguía siendo pequeña, le pregunté: "y los cuadros?! Y todo lo que estaba?!", y me contesta: "Por qué no te ocupas de tus cosas?, que el deber de los niños es de estudiar, no meterse en cosas que no le interesan".
\end{abstract}

¿No es esto acaso una clara ejemplificación de lo aquello compartido por Deleuze y Guattari (1994, p. 81) cuando sostienen que la maestra, más que comunicar "informaciones", impone al niño coordenadas semióticas para responderle? $\mathrm{O}$, mejor dicho, que si bien las palabras no son herramientas, "[...] a los niños se les da lenguaje, plumas y cuadernos como se dan palas y picos a los obreros".

De esta manera, consideramos que el hilo de la trama perteneciente a estos relatos de algún modo daría cuenta de una configuración específica, de un cierto habitus específico ligados a la educación y a la pedagogía que, al día de hoy, revistan de gran importancia: nos referimos así a la importancia de la intervención educativa en relación con los modos de configuración y producción de subjetividad que se proponen o afirman tácitamente por medio de dichas intervenciones. Por otra parte, también provocan el habla institucional en torno a los maestros como simples agentes de un Estado de facto, "ajustados" a la norma y contribuyendo a 
través de sus actos a consolidar un modelo de gestión institucional de exclusión. Por último, podría abordarse a su vez el aprendizaje e interiorización por parte de estos alumnos, de ciertos atributos y comportamientos que llevaban, muchas veces, a habitar el tiempo escolar lo menos "dolorosamente" posible. Lo anterior, aún, sin tener demasiada - o nula - comprensión de lo que sucedía en términos políticos ni históricos, pero sí en cambio, por medio de su experimentación en la ardua tarea de enfrentar el "día a día" en un aula, cuando la posibilidad de avizorar a aquel "otro" distinto o diferente se encontraba obturada.

Aquellos "auto-aprendizajes" por parte de los alumnos quizá puedan ser leídos como pruebas tangibles de lo que debía de hacerse en la vida áulica para vivir y sobre vivir en el medio escolar. Siguiendo a Jackson (1999), Perrenoud (2001, p. 14) enumera una serie de conocimientos implícitos, los cuales contribuyen a sobre-vivir en la escuela. Es por esto que aprendemos "[...] a ser evaluados de la forma que mejor sirva a nuestros intereses y proteja de la mejor manera nuestra tranquilidad [...]".

Es por eso que consideramos que: ya sea en aquella "resignación" ante la falta de interés y ante la negativa para cooperar en el intento de "salvación" del busto de Evita; entendida quizá más como un "impulso" de interiorización - y preservación de los propios efectos de sujetamiento a las normas escolares, que como acto político de un niño y que ahora dejaban no sólo de estar vigentes, sino que también eran necesarios negarlos para subsistir; o en el "oportunismo" representado por el moño negro, que oficiaba como puerta de entrada al buen trato o al desprecio escolar y paterno, según su modo de visibilidad o invisibilidad; ambos ejemplos dan cuenta de la tensión en relación con los modelos distintos de autoridad y de los mecanismos de incorporación de lo normativo, en donde, quizá en estos casos, la escuela, más que representar la paideia en donde contribuir a forjar al sujeto ético en contexto de aprendizaje, representaba el botín de lucha en donde afirmar la propia sobrevivencia y conveniencia del mundo de los adultos.

\section{Educación y crisis (2001)}

[...] piden pan, no le dan, piden queso le dan hueso y le cortan el pesquezo [...].

A partir de los casi más de 10 años transcurridos de la gran crisis del 2001, hay una suerte de consenso generalizado proveniente de diversas investigaciones sociales que expresan que: fuera del plano estrictamente económico, dicha crisis necesita aún ser asumida como parte de una revisión crítica de los valores colectivos y significaciones institucionales que dieron cuenta de la acción de muchas organizaciones, 
por intentar preservar lo poco o mucho que quedaba de su propia identidad. Esto último, en un contexto culmine de negación y de rechazo hacia lo colectivo y lo identitario. Aquella situación de alto riesgo para la genealogía - o vida - institucional, padecida en nuestro país, también impactó de lleno en la mayoría de las instituciones educativas vinculadas con nuestra comunidad.

De lo anterior se sigue que la educación escolarizada en aquella coyuntura efectivamente ha implicado una particular simbolización de ciertas cuestiones vinculadas con el poder, con la enseñanza, el aprendizaje y el reconocimiento del otro en la ardua tarea de transitar cotidianamente la vida institucional. De alguna manera, parafraseando a Robert Castel, podríamos pensar que la sociología comienza su trabajo cuando se comprende que el mundo social es sumamente duro y que la historia de la humanidad está hecha más "de pruebas dolorosas que de porvenires floridos" (CASTEL, 2010, p. 54).

Es por eso que una mirada histórica es inevitable para cualquier análisis tendiente a comprender lo que ocurre aquí y ahora. Esto implicaría analizar si las diversas situaciones adversas padecidas en aquella coyuntura sirvieron (o no) como puerta de entrada a la emergencia de otras prácticas educativas e institucionales, en el marco de la escuela en nuestra comunidad.

Pensamos que la fragmentación política y social tuvo también su re-traducción en el espacio educativo y relacional de nuestras instituciones. De alguna manera, podría sostenerse que la crisis institucional a nivel nacional, coincidió - o tuvo su correlato lógico - en la vida institucional, política y económica de todas ellas. De esta manera, entre pujas e internas políticas, la educación estatal fue objeto de severas crisis que llevaron a desestabilizar y poner en riesgo su "gubernamentalidad" e historia institucional.

Si en términos institucionales tuviéramos que resumir aquello acontecido, podríamos sostener que fundamentalmente se trató de un deterioro en donde se vieron interrumpidos los procesos instituyentes, que son aquellos que garantizan la vida y la continuidad de toda institución. Una realidad "deteriorada", de todas formas, aún podía seguir dando cuenta de una estructura capaz de permanecer y continuar, a pesar de la crisis, pero pagando el costo de una fragilización institucional progresiva.

En Argentina (inmediatamente tras el estallido social del 2001) podríamos decir que casi literalmente, al menos por un año, el país quedó prácticamente "vaciado", tanto de dinero, como de una extensa masa crítica de jóvenes y no tan jóvenes quienes, acorralados, decidieron forzosamente intentar buscar un destino en otros países. ${ }^{4}$ En este sentido es que hemos tratado de fundamentar, una y otra vez, que algunas corrientes educativas y discursos organizacionales posmodernos, que 
antes caían rendidos ante la seducción y acción del mercado y que promovían una subjetivación ahistórica del sujeto, enmudecieron ante la impostergable interpelación del sufrimiento del prójimo que se mostraba abatido y silenciado.

La crisis había arrasado con las esperanzas de gran parte de la sociedad al igual que una catástrofe natural lo hace con sus fuerzas equivalentes. La diferencia que podríamos establecer a partir de esta suerte de comparación físico-simbólica es que los pueblos y las personas suelen compadecerse o conmoverse, generalmente, más ante un "desastre" natural, "imprevisto", "fortuito", que ante las consecuencias políticas lógicas que estallan en cada país, debido a la puesta en prácticas de las propias estrategias públicas y sociales a lo largo de un determinado paso del tiempo. ${ }^{5}$

En ese contexto, no desconocimos que muchas escuelas en Argentina fueron centros de asistencialismo y, en muchos casos, también se significaron como la única presencia del Estado ante los más desfavorecidos. ${ }^{6}$ La discusión, en aquel contexto, no era cómo aprender más, sino qué hacer con toda una serie de niños desafiliados socialmente, muertos de hambre, en situación precaria y de calle y violentados también por sus contextos familiares.

Quizá un marco de referencia para contextualizar el último de los relatos, con los cuales habremos de trabajar, pueda estar vinculado en cierta forma al fragmento de la canción popular aludida en el epígrafe. Lo anterior, ya que es en la Edad Media - precisamente en España - en donde surgen los romances como género literario que expresan el sentir - y el vivir - de toda una época turbulenta. Así que tenían, por un lado, la función social de comunicar a los habitantes aquello que estaba sucediendo y, por el otro, podríamos asignarle a su vez una función educativa. Esto lo fundamentamos y pensamos a partir de que los niños, a través de rimas, juegos, arte y música, aprendían y se socializaban atenuando los efectos de una trama histórica compleja. Es por lo anterior, entonces, que consideramos que, efectivamente, dichos cancioneros contribuían a la tramitación y simbolización de lo que acontecía.

Desde los aportes de Winnicot (1993, p. 68) sabemos que el juego, libera y está vinculado a una producción de verdad, en donde el niño que juega "habita en una región que no es posible abandonar con facilidad y en la que no se admiten intrusiones". Esta suerte de introducción nos sirve para pensar, en forma homóloga, parte de lo acontecido en nuestro país con la crisis desatada allá por diciembre del 2001.

Tal como mencionamos con anterioridad, en los meses posteriores se sucedieron diversos cambios con una celeridad inimaginable, los cuales, a su vez, impactaron de lleno en los modos de relacionamiento pedagógico de lo escolar. Esto, quizás, nos confirma, una vez más, que la pedagogía no sucede o tiene lugar, exclusivamente, en las aulas. Experiencias ligadas con profundizar el reconocimiento del otro y animándose a invertir el principio de economía del mandato institucional 
reeditado bajo aquellos años del neoliberalismo, el cual giraba en torno a afianzar el interrogante bíblico del cual hablaba Levinas (1993) para señalar el punto de partida de la desaparición de la moral: ¿Acaso debo ser yo guardián de mi hermano?.

También, podríamos hacer alusión a otras experiencias que convocaban a resistir la miseria, pero, al igual que como sostuvimos con los relatos anteriores, no buscamos generalizar sino hacer visibles aquellos modos de intervención docentes que permitieron una visualización social de la tarea escolar diferente - y señalar su incidencia en la constitución de subjetividad crítica - en aquella coyuntura; ya que, como la otra cara de una misma moneda, también podrían señalarse experiencias que, en el medio de la crisis, también sirvieron para afianzar un cierto posicionamiento de clase y de demarcación social y política. En relación con esto último, podríamos hacer referencia entonces a un establecimiento educativo en donde, desafiantemente, pudo escucharse a unos padres decir en la puerta de la escuela: "Por fin ahora vamos a saber quiénes podrán seguir viajando a Europa".

Retomando nuestro propio posicionamiento como objeto de estudio en este apartado, consideramos que hubo un gran sufrimiento colectivo plasmado en los altos índices de desocupación, de miseria, pero también de tristeza y de impotencia. Sin embargo, algunos recuerdos que hoy afloran en estos hoy adolescentes de aquella (reciente) época podrían dar cuenta de la metáfora medieval de los romances, pero en el sentido de que pareciera que la escuela, a pesar de todo, pudo contribuir a mitigar - y a simbolizar -, por medio de su accionar parte de lo vivenciado:

\begin{abstract}
Mirá, lo que me acuerdo es que estábamos en segundo grado y estaba más bueno estar en la escuela que en mi casa. Mi papá no se levantaba de la cama porque estaba triste porque mi abuelo falleció de la "bronca" por quedar atrapado en el corralito. Mi mamá nos llevaba a la escuela y allí jugábamos con mis compañeros en los recreos con alguno que otro de esos billetes que parecían de juguete porque venían de muchos colores diferentes y nos hacían acordar a los de los "juegos de mesa" tipo el "estanciero"7... así que, juntábamos los que teníamos - que eran los que justo no recibían nadie - y los repartíamos entre todos para jugar. Me acuerdo que la señorita de clase estaba fascinada con el tema de Diego Torres "Color Esperanza" y cada vez que podía, lo poníamos en los actos, en las clases y la pasábamos cantándolo... a mí me gustaba, pero después de tanto cantarlo nos aburría... pero como "todos" en la escuela se ponían tan contentos al escucharlo, lo volvíamos a cantar en todos los actos de nuevo.
\end{abstract}

En este sentido, podríamos afirmar que el acontecimiento histórico puede contribuir a fundar o a instaurar las bases para que un nuevo sujeto pueda emerger de la crisis; $y$, a partir de ese momento, los juegos se significarán como capaces de dar cuenta de algunas verdades, como así también de ciertos efectos subjetivos y estéticos impulsados colectivamente por el canto de nuevas canciones. En esta instancia, preferimos dejar de lado la discusión en torno al valor estético o musical de las mismas, para centrarnos en esta suerte de "función" social que aún lleva a que, cuando 
son escuchadas, hasta el día de hoy, conserve todo el sabor de una época asociada a una plena recuperación. ${ }^{8}$ Porque lo que en todo caso cobró vital importancia en esa coyuntura - y en ese ejemplo particular - fue precisamente el acontecimiento como posibilidad de repensar y repensarse a través del canto colectivo.

Aquí es donde entonces, en ese contexto, surgen nuevas propuestas pedagógicas provenientes de otros campos, los cuales solían presentarse como "desestimados" o, por lo menos, menos importantes en relación con aquellos que servían para dar cuenta de los conocimientos oficialmente legitimados, previamente a esa crisis que tuvo lugar:

\begin{abstract}
Yo estaba en sexto grado y la verdad es que si era por mí, hubiera dejado la escuela para ir a ayudar a mi papá a trabajar en su negocio porque lo veía medio triste. Por suerte no me dejaron y en la escuela nos hablaban y alentaban para que no hiciéramos eso porque había otros chicos que les pasaba lo mismo que a mí. En la escuela nos ayudaron mucho, íbamos seguido y a diferencia de antes, tratábamos de no faltar porque "por lo menos" la pasábamos bien y aprendíamos... se organizaban charlas, había deportes, talleres... lo que más recuerdo es que íbamos a un ciclo de cine con un televisor que tiempo atrás habían donado en la escuela y que le habían puesto (al ciclo) "cine con bizcocho", porque había que ir con una bolsita con bizcochos para comer y compartir... Mi mamá se juntaba con otras madres y, en vez de comprar bizcochos, hacían tortas fritas, las llevábamos, y de paso también las vendíamos a la tarde durante los días de invierno por el barrio, cuando regresábamos de la escuela.
\end{abstract}

Podríamos decir, entonces, que el campo educativo desde la óptica del presente relato, el cual, insistimos, no es utilizado aquí para generalizar sino para poner en palabras solo una parte (representativa) de un complejo rompecabezas, pudo conformarse como un espacio de socialización por medio de aprendizajes fundados - y fundantes - a partir de aquel nuevo escenario social, político, económico y educativo, inherente a aquella verdad que se tejía y desprendía de aquel acontecimiento. Badiou (1995, p. 125) expresa que:

La fidelidad al acontecimiento es ruptura real (pensada y practicada) en el orden propio en el que el acontecimiento ha tenido lugar (político, amoroso, artístico, científico). Se llama verdad (una verdad) al proceso real de una fidelidad a un acontecimiento. Aquello que esta fidelidad produce en la situación.

Y continúa afirmando que "se llama 'sujeto' al soporte de una fidelidad, luego, al soporte de un proceso de verdad. El sujeto no preexiste para nada a un proceso. El es absolutamente inexistente en la situación 'antes' del acontecimiento. Se dirá que el proceso de verdad induce un sujeto" (ibíd., p. 126). Y para nosotros, es precisamente este nuevo sujeto quien pudo surgir en el seno mismo de ese acontecimiento expresado en un proceso de verdad: fidelidad a los afectos, fidelidad al valor del conocimiento; incluido aquí todos aquellos que alguna vez fueron erradicados 
del ámbito del saber dominante; fidelidad al valor de la educación junto con su capacidad de devolver la re-significación de las palabras vaciadas ahora hechas acciones; al valor del juego como producción de verdades; $y$, sobre todas las cosas, la fidelidad de volver a ser porque se es y no porque se tiene. Si hay algún proceso de verdad para enunciar y compartir, quizá éste último sea el más importante.

\section{Consideraciones finales}

Intentar reflexionar a partir de algunas coyunturas históricas específicas, desde las huellas aún perdurables que se reconstruyen a partir de los relatos aquí presentados, nos ha permitido hablar acerca de las formas de habitar la escuela como así también del ser alumno y del ser docente, a lo largo de la historia.

A fin de articular parte del contenido de los diversos relatos, quizás creamos que podría ser aquí simplificado, aludiendo a una división comprendida a través de dos grandes relatos o divisiones simbólicas: estos relatos nos hablan de la educación o bien como parte de un proceso de identificación con el poder; y la educación entendida como un proceso que alienta y promueve procesos de des-identificación con el mismo. Sólo a los efectos didácticos en la presentación de este esquema, quizás también se nos permita sostener en forma provisoria que la historia en "sí misma"; junto con la de los sujetos encargados de contribuir a la historia de la pedagogía y de la educación pueda ser simplificada bajo esta humilde - pero significativa - taxonomía. Aquellas corrientes, autores y sujetos que, a lo largo de la historia, intentaron con su discurso asumir e introducir una diferencia crítica en la construcción y modos de subjetivación escolares; esto es, a través de la búsqueda de una construcción de una posición subjetiva, afiliando al prójimo en un proyecto de emancipación política, social y espiritual del mismo; o aquellas corrientes o posiciones subjetivas y teóricas que lo ubicaban menos como sujeto parte de una comunicación que como objeto de información con derecho a tecnificar, entrenar, disciplinar, expropiar, extraer, desconocer, dañar, cosificar o alienar.

En la introducción, habíamos hecho referencia a que el núcleo fuerte de los relatos tendría que ver con aquello persistente y perdurable. Entonces podría decirse que, por un lado, el hilo conductor de los relatos compartidos pasa o tendría que ver exclusivamente con la restricción o pérdida de la palabra. Si así fuera, a pesar de que no estaríamos faltando a la verdad, se estaría dejando a un costado del camino uno de los aspectos más relevantes que emergieron a la luz del trabajo investigativo.

Ya que, como hemos intentado dar cuenta, más allá de las diferencias y las limitaciones en cada coyuntura histórica abordada, la fuerza crucial de los relatos han residido en su capacidad para, aún al día de hoy, continuar dando cuenta de 
ciertos soportes y procesos de verdades. A su vez, también de brindarnos herramientas conceptuales para significar e intentar comprender las distintas formas y maneras de habitar la escuela: ya sea en el marco de un adulto que, a pesar del paso del tiempo y ya asomado a sus cuarenta años, persiste y deja vivir en él la fidelidad forjada por la promesa de transformación social de una escuela que fue rifada en su punto de máximo esplendor académico por la dictadura; o de aquellos adultos hoy ya mayores quienes no pueden dejar de recordar sin dolor el busto de Evita - ya sea por tener que negarlo o afirmarlo en la propia escuela; o como también aquellos incipientes jóvenes quienes recién empiezan a transitar su vida y que algún día tendrán que dar cuenta acerca de qué pudieron hacer con aquella fidelidad experimentada en el seno mismo de la escuela; y qué hicimos también nosotros en el presente, para por lo menos intentar asegurar que la educación pueda devenir como aquella paideia, en donde los procesos de verdades sean el soporte y andamiaje de nuestra propia existencia, a lo largo de la historia.

\section{Notas}

1. La Biblioteca Popular Constancio C. Vigil fue una de las experiencias más importantes de educación y expresión popular de América Latina, devastada en 1977 por los militares En una primera instancia (1953), nace la biblioteca Constancio C. Vigil como una subcomisión de la Sociedad vecinal; en 1956 realiza su primera rifa y el dinero juntado es destinado a la compra de libros y de material bibliográfico y pedagógico. La rifa alcanza un éxito sin precedentes y se extiende a gran parte del país, con una red mensual de más de 20.000 socios. Años más tarde se crean el Instituto secundario, el primario y el Jardín de infantes con un enfoque pluralista, crítico e inclusivo a través del arte, la música, el teatro y la literatura.

2. La organización y movimiento aludido es "Montoneros"; la cual se identificaba con la izquierda peronista.

3. Personaje ficticio correspondiente a la serie La familia Adams.

4. El "corralito" expresó el dispositivo con el cual los bancos de todo el país restringieron las extracciones de dinero en efectivo por parte de sus clientes para, en forma posterior, directamente pasar a confiscarlos hasta tanto y en cuanto se accediera a un plan de devolución vía judicial, arreglos con el propio banco, o vía Bonos del Tesoro. En los meses previos a las disposiciones nacionales que otorgaban el derecho de extracción para personas enfermas o de edad muy avanzada, diversas personas murieron en el intento por recuperar sus ahorros.

5. Aquello que queremos señalar tiene que ver con el carácter de "fatalidad incierta" y de angustia que provocan los efectos de las catástrofes naturales. Ahora bien, tampoco podemos desconocer que, muchos de esos efectos también podrían verse "amenizados" si se tomaran ciertos recaudos, los cuales, aunque insuficientes ante el poder destructivo de la naturaleza, sí en cambio permitirían amplificar el coeficiente e sobrevivientes. Para esta temática, confrontar: Hartman y Gregory (2006).

6. Algunos autores que han abordado parte de estas temáticas (cf. bibliografía final): Duschatzky y Birgnin (2001); Tenti Fanfani (2007); Palamidessi, Suasnábar y Galarza (2007); Gvirtz, Grimberg y Abregú (2007); Skliar y Larrosa (2009).

7. Aquí se está haciendo referencia a las cuasi-monedas, con las cuales, a lo largo de un lapso considerable de tiempo, los diferentes gobiernos provinciales comenzaron a emitir como parte de pago de los sueldos de sus empleados. De esa forma, en la ciudad hasta podían "convivir" con el "legítimo"

Educ. Soc., Campinas, v. 35, n. 127, p. 511-527, abr.-jun. 2014 
peso diferentes emisiones bancarias que oficiaban de sustitutos para cubrir la función de herramienta monetaria del peso: éstas eran denominadas Lecop; Patacon; Lecor; Quebracho; Bocade; entre otros.

8. En este sentido, quisiéramos subrayar la capacidad y el poder de la música para generar conciencia crítica a lo largo de nuestra historia, ya que, a partir de la misma, se pueden observar que profundas transformaciones sociales, políticas y religiosas han tenido sus propias expresiones musicales. Bastaría señalar la "Marsellesa", "La Internacional", o la importancia de los cantos de Lutero, que llevaron a sostener a algún enfurecido clérigo antireformista que todos sus cantos habían "envenenado" más almas que todos sus libros y todos sus discursos; a su vez, sabemos de la importancia de la música para los griegos en sus sistemas de enseñanzas y ya situándonos a mediados del siglo XX, la marca que dejó en toda una generación la música del filme sobre Sacco y Vanzetti.

\section{Referencias}

ARENDT, H. Hombres en tiempos de oscuridad. Buenos Aires: Gedisa, 2008.

BADIOU, A. La ética. In: Batallas éticas. Buenos Aires: Nueva Visión, 1995.

CASTEL, R. El ascenso de las incertidumbres. Trabajo, protecciones, estatuto del individuo. Buenos Aires: FCE, 2010.

DELEUZE, G.; GUATTARI, F. Mil mesetas. Valencia: Pre-Textos, 1994.

DUSCHATZKY, S.; BIRGNIN, A. ¿Dónde está la escuela? Ensayos sobre la gestión institucional en tiempos de turbulencia. Buenos Aires: Flacso, 1991.

FILLOUX, J.C. Epistemología, ética y ciencias de la educación. Córdoba: Encuentro, 2008.

GIDDENS, A. Hermenéutica y teoría social. In: GIDDENS, A. Profiles and critiques in social theory. Berkeley: University of California, 1982. cap. 1 (Trad. de la Cátedra de Epistemología, Facultad de Psicología, Universidad de Rosario).

GVIRTZ, S.; GRIMBERG, S.; ABREGÚ, V. La educación ayer, hoy y mañana. El ABC de la Pedagogía. Buenos Aires: Aique, 2007.

HARTMAN, C.; GREGORY, S. (Ed.). There is no such thing as a natural disaster: race, class and hurricane Katrina. New York: Routledge, 2006.

JACKSON, P. La vida en las aulas. Madrid: Morata, 1999.

KAUFMANN, C.; DOVAL, D. Libros aprobados; Libros prohibidos; Libros recomendados: Argentina 1976-1982. In: KAUFMANN, C.; DOVAL, D. Una pedagogía de la renuncia: el perennialismo en la Argentina 1976-1983. Entre Ríos: Universidad Nacional de Entre Rios, 1997. p. 45-70.

LEVINAS, E. Entre nosotros. Ensayos para pensar en otro. Valencia: Pre Textos, 1993. PALAMIDESSI, M.; SUASNÁBAR, C.; GALARZA, D. Educación, conocimiento y política. Argentina, 1983-2003. Buenos Aires: Flacso-Manantial, 2007. 
PERRENOUD, P. La construcción del éxito y del fracaso escolar: hacia un análisis del éxito, del fracaso y de las desigualdades como realidades construidas por el sistema escolar. La Coruña: Paidéia; Madrid: Morata, 2001.

RICOEUR, P. Educación y política: de la historia personal a la comunión de libertades. Buenos Aires: Prometeo, 2009.

SKLIAR, C.; LARROSA, J. Experiencia y alteridad en educación Buenos Aires: Homo Sapiens/Flacso, 2009.

TEDESCO, J.C. Educación y sociedad en la Argentina (1880-1945). Buenos Aires: Solar, 1993.

TEDESCO, J.C. Elementos para una sociología del currículum escolar en Argentina. In: TEDESCO, J.C.; BRASLAVSKY, C.; CARCIOFI, R. El proyecto educativo autoritario: Argentina 1976-1982. Buenos Aires: Miño y Dávila, 1987. p. 17-73.

TENTI FANFANI, E. El oficio de docente. Vocación, trabajo y profesión en el siglo XXI. Buenos Aires: Siglo XXI, 2007.

WINNICOTT, D. Realidad y juego. Barcelona: Gedisa, 1993.

Recebido em 31 de janeiro de 2013.

Aprovado em 18 de fevereiro de 2014. 